\title{
Mining Mid-level Features for Image Classification
}

\author{
Basura Fernando • Elisa Fromont • \\ Tinne Tuytelaars
}

Received: date / Accepted: date

\begin{abstract}
Mid-level or semi-local features learnt using class-level information are potentially more distinctive than the traditional low-level local features constructed in a purely bottom-up fashion. At the same time they preserve some of the robustness properties with respect to occlusions and image clutter. In this paper we propose a new and effective scheme for extracting mid-level features for image classification, based on relevant pattern mining. In particular, we mine relevant patterns of local compositions of densely sampled low-level features. We refer to the new set of obtained patterns as Frequent Local Histograms or FLHs. During this process, we pay special attention to keeping all the local histogram information and to selecting the most relevant reduced set of FLH patterns for classification. The careful choice of the visual primitives and an extension to exploit both local and global spatial information allow us to build powerful bag-of-FLH-based image representations. We show that these $b a g$-of-FLHs are more discriminative than traditional bag-of-words and yield state-of-the-art results on various image classification benchmarks, including Pascal VOC.
\end{abstract}

Keywords Frequent itemset mining · Image classification · Discriminative patterns · Mid-level features.

\section{Introduction}

Vector quantized local features, be it densely sampled or extracted from interest points, have a proven track record in vision. They are the default image representation for a wide variety of applications ranging from image retrieval

B. Fernando, T. Tuytelaars

KU Leuven, ESAT-PSI, iMinds Belgium

E. Fromont

Université de Lyon, Université de St-Etienne F-42000,

UMR CNRS 5516, Laboratoire Hubert-Curien, France 
to image classification. Although early papers presented them as a kind of automatically discovered object parts (e.g. wheels of airplanes) [42], they have, in practice, only limited semantic meaning in spite of what the name visual words suggests.

Based on this observation, some recent works $[6,14,22,26,39,41]$ have looked into the construction of more distinctive, mid-level features (sometimes also referred to as semi-local features or parts). Some of them operate in a strongly supervised setting (e.g. poselets [5]), while others use only weak supervision (i.e. no annotations at the parts-level, only at class-level), e.g. hyperfeatures [1], discriminative patches [41] or blocks that shout [22]. Here we focus on the weakly supervised case (which, by the way, has been shown to outperform the strongly supervised case [22]).

A first set of methods (e.g. [6,14,22]) starts from the same description that proved its value for local features, building on robust gradient-based representations such as HoG [13]. The main difference lies in the fact that for mid-level features, typically larger (or actually, more detailed) patches are used compared to those used traditionally for local features. This makes them more informative, so potentially more distinctive. However, this also makes them more sensitive to misalignments, deformations, occlusions, parts not falling entirely on a single surface or object, etc. Simply clustering such larger patches, as done for local features, does not work very well under these circumstances. Besides, existing iterative clustering approaches are not guaranteed to converge to the global optimum and depend on the initial seeds. If the deformations are big, it is not clear that the resulting patches in the clusters are indeed uniform in appearance (as we also observed in our experiments). Instead, typical variations in appearance of the mid-level features need to be learned, e.g. using exemplar SVMs as done in $[22,41]$. However, these methods also start with an initial clustering or nearest neighbour search, which again limits them to cases with not too big deformations. Object parts close to an object boundary, (self)occlusion or orientation discontinuity are not likely to be found with these methods.

Moreover, HoG patches are rigid. For small patches (local features), this is often a reasonable approximation, as shown by the success of SIFT [29]. However, with the patches becoming larger, the rigidity of HoG makes the features very specific. They seem to lack the necessary flexibility needed to cope with the observed deformations, be it due to viewpoint changes or due to within-class variability.

A second set of methods (e.g. $[1,40])$ builds on more robust representations typically used for global object or scene descriptions, such as bag-of-words [12] or Fisher Vectors [33]. Representing a mid-level feature based on the distribution of the local features it contains, brings the necessary flexibility to cope with deformations. In the work of Agarwal and Triggs [1], mid-level features are represented with local bag-of-words, which are then clustered to obtain a set of mid-level vector-quantized features. Simonyan et al. [40] do the same for Fisher Vectors, and further add a global discriminative dimensionality reduction step. In both cases, the link between the mid-level feature and the 
low-level features composing it, is lost in the final representation. Moreover, for neither of these methods the construction of mid-level features is selective: if some low-level features inside the mid-level patch fall on the background or turn out to be unstable, they cannot be ignored, adding noise to the overall process.

Finally, some methods (e.g. $[27,28,10,37,49,51])$ have looked at constructing mid-level features by combining multiple low-level features. However, this quickly brings combinatorial problems, and seemed not that successful in practice. Moreover, combinations have been limited to relatively few elements (usually pairs or triplets).

In this paper, we propose a new method to extract mid-level features, building on the extensive pattern mining literature and expertise. Mid-level features are constructed from local bag-of-words (LBOW, as e.g. used in $[1,35,43,53]$ ) representing the distribution of local features in a semi-local neighbourhood. However, we do not keep the full bag-of-words, but select subsets of low-level features, that are optimal in terms of discriminativity, representativity, and non-redundancy. We refer to the resulting mid-level features as Frequent Local Histograms or FLHs. Each FLH is composed of a set of low-level visual words that are spatially close. Compositions are not restricted to pairs or triplets, as in $[10,27,28,37]$, but can easily contain ten or more elements. Since we focus only on the number of these elements within a local image area, ignoring their spatial configuration, the resulting representation is very flexible and can cope well with various types of image deformations, occlusions and image clutter. Moreover, being constructed from standard low-level features (visual words), they can be indexed efficiently, as we show in [19]. From all sets of visual words that co-occur frequently, we select the final set of FLHs based on their discriminativity for the classification task at hand, their non-redundancy as well as their representativity. While the data mining techniques we apply are rather standard, we adapt them to our specific setting aiming at mid-level visual features that yield good results on various image classification tasks.

Even though frequent itemset mining techniques and variants thereof are well-established in the data-mining community [3,45], they are, to date, not commonly used in state-of-the-art image classification methods. This is surprising, since it has been shown that these mining methods allow the construction of high-level sets of compound features which can, in many cases, capture more discriminative information [9]. Nevertheless, most attempts so far applying pattern mining to image classification $[31,50,35,53]$ were not able to demonstrate competitive results on standard datasets. Here, we propose an effective scheme for applying pattern mining to image classification by adapting the generic pattern mining tools to the specific context of visual features extracted from images. We provide an extensive analysis of parameter selection and relevant pattern mining. We compare several spatial pyramid schemes which capture both local and global spatial information with and without data mining, and we highlight some advantages of mining-based approaches that capture local spatial information compared to non-mining methods. 

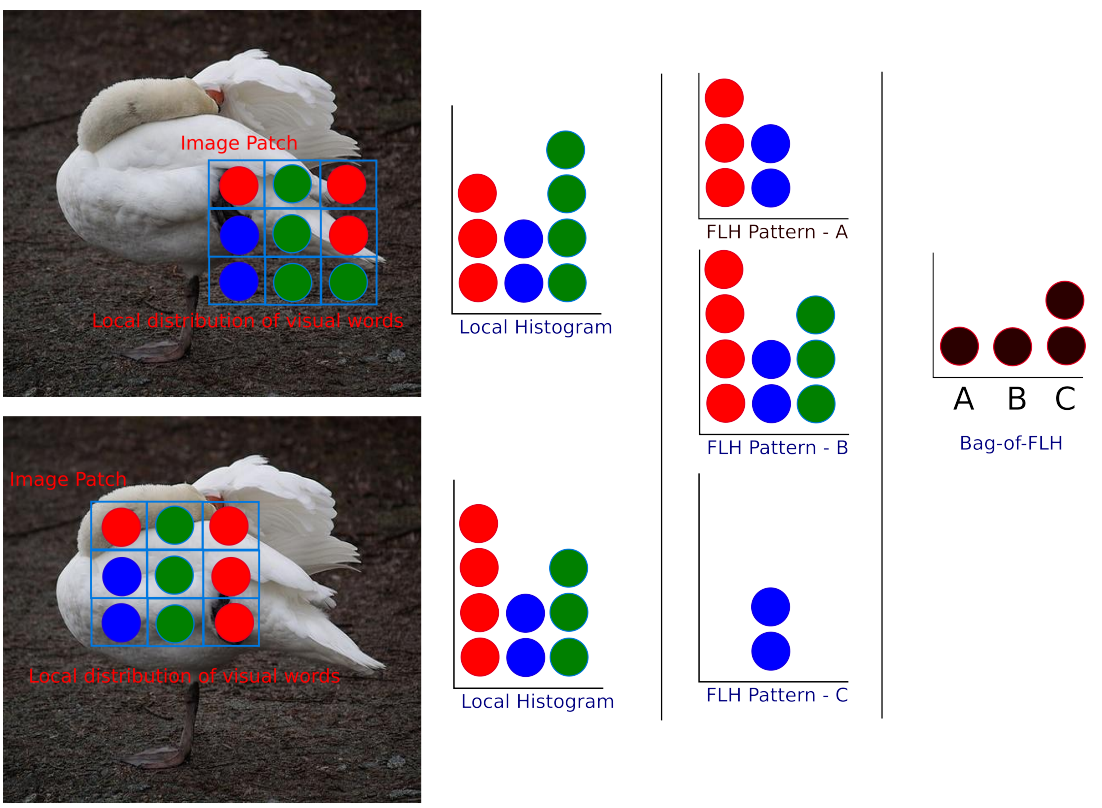

Bag-of-FLH

Visual Words $=$

Fig. 1: FLH mining and image representation process. First dense SIFT descriptors are extracted. Each descriptor is assigned to a visual word (hard assignment). For each dense descriptor, its $\mathrm{K}$ spatial nearest neighbors are selected (in practice, we use all local descriptors within a square $n \times n$ neighbourhood). From these descriptors a local bag-of-words (LBOW) representation is created for each dense point. Then we mine for the most frequent local histograms from the entire dataset. These frequent local histograms are known as FLH patterns or just FLHs. Afterwards, using a post processing step, we select the most suitable set of FLHs for image classification. We encode the LBOWs in an image using these relevant FLH patterns (but note that some of the LBOWs won't be captured by any of the selected FLH patterns). Finally by counting how many times each FLH pattern is used to encode an image, we create a bag-of-FLHs representation.

The composition of the following three aspects makes our method different from earlier approaches that apply pattern mining to bag-of-visual-words (noted BOW in the rest of the paper): i) we start from local BOW (LBOW) to capture local information, ii) we take particular care in loosing as few information as possible during the conversion step necessary to transform a histogram (LBOW) into a suitable input for a mining algorithm (known as a transaction) and iii) we carefully but automatically select the relevant and non redundant patterns that will be used in our classification step. The bag-of-FLHs creation process is shown and explained in the caption of Fig. 1. 
This paper extends our earlier work [18] with more details about our motivations and related works as well as more extensive experimental validation. In particular, we added experiments comparing our approach to non-mining ones and combining FLH and Fisher vectors, which led to very good results.

The rest of the paper is organized as follows. First, we review related work in section 2. Section 3 provides details on the construction of relevant FLHs and shows how they can be used for image classification. In Section 4 we show how local and global spatial information can be combined. Section 5 describes the experimental validation, demonstrating the power of our method for challenging image classification problems. In Section 6 we compare FLH with state-of-the-art methods. Section 7 concludes the paper.

\section{Related work}

Frequent pattern mining techniques have been used to tackle a variety of computer vision problems, including image classification $[23,31,53,54]$, action recognition [20,34], scene understanding [50], object recognition and objectpart detection [35]. Apart from the application, these methods mostly differ in the image representation used, the way they convert the original image representation into a transactional description suitable for pattern mining techniques and the way they select relevant or discriminative patterns. We therefore organize our discussion on related works along these three axes. We end with a discussion of other methods for extracting mid-level features.

Image representations: A standard image representation nowadays is the bagof-visual words [12]. The BOW can be computed either globally or locally in a neighborhood around an interest point (LBOW). In the context of pattern mining-based image classification, local bag-of-words are usually preferred (e.g. in $[23,34,35,43,53])$, since they result in sparse representations, a better signal-to-noise ratio, an increased robustness to image clutter and some low level spatial information (proximity). Spatial configuration mining based on LBOW was first shown by Quack et al. [35], although they did not use these configurations for classification. Perhaps, the distinctive feature configurations mined in their work were too specific, making them less suited for image classification. Secondly, they only relied on interest points to create object specific transactions. In contrast, FLH uses dense sampling (which captures a larger amount of statistics) and relevant pattern mining to find not just frequent but rather discriminative image representations. More structured patterns such as sequences and graphs capturing the spatial distribution of visual words have been used by [31], while [52] uses boosting on top of binary sets of visual words discovered by pattern mining. Gilbert et al. [20] have applied itemset mining to action recognition using rather primitive features like corners, while in [54] high level features such as attributes [16] are successfully used with mining techniques. In [50], Yao et al. present a structured image representation called 
group-lets. To find discriminative group-lets, they mine for class-based association rules. Association rule learning [2] is used for discovering relations between variables using different measures of interestingness. Class-based association rules are used to find relations between itemsets and class variables. Itemsets that are highly associated with class variables are selected as patterns.

However, none of the above representations took a particular care both in designing a suitable encoding to effectively use pattern mining for image classification and, in designing a dedicated post-processing step to obtain stateof-the-art classification results on standard image classification datasets.

Transforming bags to transactions: Most existing mining methods simply use individual visual words as items in a transaction. Transactions are created in such a way that if a visual word is present in the histogram, then it is also present in the transaction (i.e. itemset). Information on the exact frequency, i.e. how often the visual word occurred in the LBOW, is lost in this process. In [23], Kim et al. use a new representation called Bag-to-set (B2S) to transform a histogram into a transactional form without losing information. In this approach, each bin of the histogram is converted into a sequence of binary digits. The length of this sequence is given by the largest value that a specific bin can take. For example, if the maximum value for the first bin of a set of histograms is 5 and in a particular histogram, this first bin has the value 3 , its B2S representation will be [11100] (the length of the sequence is 5 and only the 3 first values are "true"). Afterwards, B2S concatenates all the sequences (from all the bins in the histogram), transforming the histogram into a binary sequence that can be regarded as a "transaction" by a traditional pattern miner. The B2S representation is, to our knowledge, the only unsupervised effort to explicitly avoid information loss in the conversion to transactions. However the mining phase might generate artificial visual patterns (ghost patterns) that do not exist in the image database (see Fig. 2 for an example of such ghost patterns). For example, this encoding implies that when a histogram bit has some particular value $x$, all the values lower than $x$ are also true at the same time in the binary encoding. This could result in wrong possible matching as shown for pattern c(1) in Fig. 2. Besides, a pattern could incorporate parts of a bin description (for example the first and last "1" without the middle ones) that would have no "real" meaning according to the original encoding. These ghost patterns hinder the performance of the patterns constructed using the B2S method.

Alternatively, one could give each visual word a weight depending on how many times it appears in the histogram and apply weighted itemset mining [55]. However, this method only postpones the loss of information as it then simply sums all the itemset weights to discover patterns. FLH, as proposed in this paper, avoids information loss without generating unexisting patterns, as the number of occurrences of a visual word must match exactly.

Mining relevant patterns: Frequent patterns can be more discriminative than individual features (e.g. visual words), since a pattern is a combination of sev- 

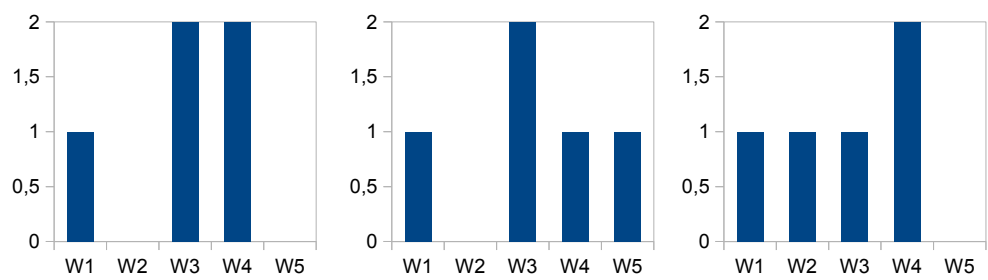

(a) Examples of local histograms (LBOW).
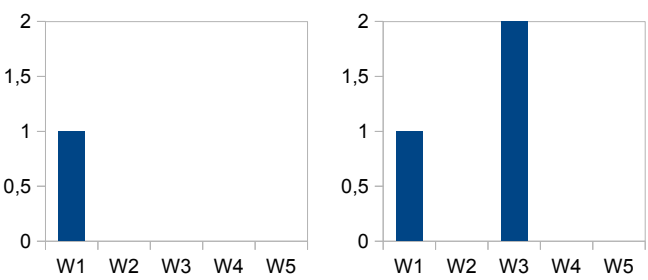

(b) Examples of FLH patterns extracted from the above LBOW
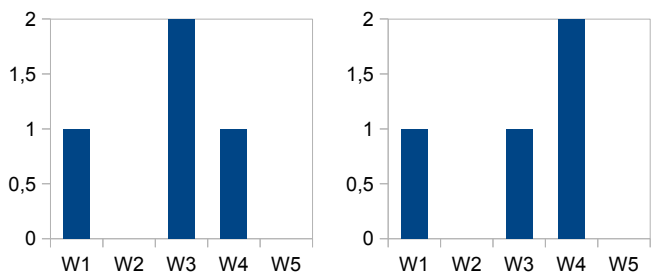

(c) Examples of B2S patterns extracted from the above LBOW
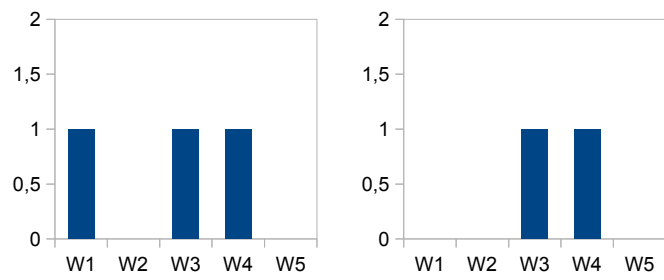

(d) Ex of frequent patterns extracted from the above binarized LBOW

Fig. 2: Three different transaction creation methods and resulting patterns. For all the figures, the $\mathrm{X}$ axis shows the different visual words and the $\mathrm{Y}$ axis gives the value of the histogram bin for each visual words. (a) represents some local histograms. (b) shows some resulting FLH patterns extracted from the histograms in (a). (c) shows some patterns extracted using the B2S approach. (d) shows some patterns extracted from binarized histograms. Both FLH and B2S do not lose any information during the transaction construction stage. However, B2S generates some patterns that would be mapped to non existing data which we call ghost patterns. For example, (c1), the first B2S pattern (Fig. (c) first pattern) would be mapped to the first and second local histograms ((a1) and (a2)). Nevertheless, (a1) should not be mapped to (c1) as the word frequencies of W4 do not match. 
eral primitives and therefore likely to capture more distinctive information. However, when using local bag-of-words to create transactions as we plan to do, each source (image) generates multiple transactions and a pattern that is found only in a relatively small number of images can still be frequent if it appears in really high numbers within this small set of images. Applying standard relevant pattern discovery methods under these circumstances, as done in $[20,35,50,53]$, may not be the best strategy. Most of the methods that use pattern mining in the context of image classification are restricted to standard class-based association rules [2], standard discriminative pattern mining approaches $[20,25,35,50,53]$ or other supervised methods such as boosting to find interesting patterns [31,52]. In [53], Yuan et al. present a visual pattern mining algorithm based on a likelihood ratio test to find relevant patterns in an unsupervised manner. None of these works considers the issue of repetitive structures in images, causing frequent yet not representative patterns. Therefore, for a pattern to be useful it should be both discriminative and representative.

Mid-level feature extraction: As mentioned already in the introduction, other methods have been proposed to extract mid-level features using compositions of low-level features. However, most of them are limited to the use of pairs or triplets of features $[10,27,28,37]$. Only a few have used higher-order statistics (co-occurrence of visual words), albeit on a single image [49] or pairs of images [51] only. Unlike pattern mining, they do not exploit database-wide statistics.

Early work on extracting mid-level features are the hyper-features of Agarwal and Triggs [1]. Like us, they start from local-bag-of-words but cluster them recursively (in a hierarchical fashion) to find a new set of spatial features called hyper-features. Then they represent each image as a bag-of-hyper-features. In our work, FLH patterns are constructed from local histograms in which subhistograms are mined as patterns instead of clustering entire local histograms. While the approach of [1] also captures larger patterns, it does not have the same flexibility in local geometry as our scheme.

Boureau et al. [6] have proposed to construct macro-features by jointly encoding a small neighbourhood of SIFT descriptors from a $2 \times 2$ or $3 \times 3$ square. As in the case of hyper-features, this method cannot selectively add a patch (SIFT descriptor) to the macro-feature. In contrast, in our FLH approach, each patch in the local neighborhood is selected to be included in a pattern only if it satisfies certain criteria (such as frequency, discriminativity, redundancy etc.). As a result, and in contrast to [6], FLH patterns are robust to spatial deformations, occlusions and image clutter.

In [41], Singh et al. start with HoG templates and try to find discriminative and representative patches using a clustering and a support vector machinebased approach. Unfortunately, there is no guarantee on the cluster purity or the class-based representativeness of the clusters. Nevertheless, empirically this method manages to converge to a set of reasonable clusters. In a similar spirit, Juneja et al. [22] learn parts incrementally, starting from a single part 
occurrence with an exemplar SVM and gradually adding more examples after an alignment step. As mentioned earlier, we believe that starting from a rigid HoG representation does not allow sufficient flexibility and makes the parts too specific. We take a different approach and build more general (in the sense of more flexible) mid-level features as compositions of several local rigid SIFT features. It turns out the tradeoff between rigidity at a local scale and flexibility at a mid-level scale) is an important parameter when building midlevel features (e.g. by changing the size of the local SIFT features).

Our approach exploits the local statistics of an image while methods such as Fisher vector-based approaches [21] exploit zero, first and second order global statistics of an image $[33,8]$. Consequently, as shown in the experiments, our method is complementary to Fisher vector encoding. Recently, Simonyan et al. [40] have proposed Deep Fisher Networks, that apply the Fisher vector encoding at a semi-local scale. Integrating Fisher vectors in our framework in a similar fashion, is an interesting research direction we plan to investigate in the near future.

\section{FLH-based Image Representation and Classification}

After introducing some notations, we explain how we mine frequent local histograms (FLHs) (section 3.1). We then show how we select the most relevant set of FLHs for image classification (section 3.2) and present a suitable kernel for relevant pattern-based image classification (section 3.3).

Each image $I$ is described by a set of features $\left\{f_{i} \mid i=1 \ldots n_{I}\right\}$ and a class label $c, c \in\{1 \ldots C\}$. We assume that all the descriptors have been clustered to obtain a set of so-called visual words. Then, each key point $f_{i}$ is given a label $w_{i} \in W$ known as the visual word index. $|W|$ is the visual word dictionary size. In our approach, for each feature $f_{i}$ we compute a local histogram (also called a local bag-of-words LBOW), $\mathbf{x}_{\mathbf{i}} \in \mathbb{N}^{|W|}$ using the $K$ spatial nearest neighbours of $f_{i}$ (based on the distance between image coordinates and also including $f_{i}$ itself as a neighbour). In practice, we use all features within a local square neighbourhood of size $n \times n$ around the feature. The set of all the local histograms $\mathbf{x}_{\mathbf{i}}$ created from all images is denoted by $\Omega$.

\subsection{Frequent local histogram mining}

Items, Transactions and Frequencies: In order to avoid loss of information during the transaction creation process without generating ghost patterns, we propose the following new definition of an item. An item is defined as a pair $(w, s), w \in W$ and $s \in \mathbb{N}$, with $s$ being the frequency of the visual word $w$ in the local histogram. Note that $0<s \leq K$ and for a given image there is at most one item per histogram bin.

Next, we create the set of transactions $X$ from the set of local histograms $\Omega$. For each $\mathbf{x} \in \Omega$ there is one transaction $x$ (i.e. a set of items). This transaction 
$x$ contains all the items $\left(w_{j}, s_{j}\right)$ such that the bin corresponding to $w_{j}$ in $\mathbf{x}$ has the nonzero value $s_{j}$. A local histogram pattern is an itemset $t \subseteq \Gamma$, where $\Gamma$ represents the set of all possible items. For any local histogram pattern $t$, we define the set of transactions that include the pattern $t, X(t)=\{x \in X \mid t \subseteq x\}$. The frequency of $t$ is $|X(t)|$, also known as the support of the pattern $t$ or $\operatorname{supp}(t)$.

Frequent Local Histogram: For a given constant $T$, also known as the minimum support threshold, a local histogram pattern $t$ is frequent if $\operatorname{supp}(t) \geq T$. A pattern $t$ is said to be closed if there exists no pattern $t^{\prime}$ such that $t \subset t^{\prime}$ and $\operatorname{supp}(t)=\operatorname{supp}\left(t^{\prime}\right)$.

The set of frequent closed patterns is a compact representation of the frequent patterns (i.e we can derive all the frequent patterns from the closed frequent ones). In this work we refer to a frequent and closed local histogram pattern as a Frequent Local Histogram or FLH. $\Upsilon$ is the set of all FLHs.

FLH Mining: Given the set of transactions $X$, we can use any existing frequent mining algorithm to find the set of FLHs $\Upsilon$. What is specific to our method is that i) the input of our algorithm is a set of local histograms $\Omega$, and ii) a preprocessing step is performed building the set of transactions $X$ from the local histograms $\mathbf{x}_{\mathbf{i}}$ as described above. Items $\left(w_{k}, s_{k}\right)$ in a transaction $x \in X$ can then be regarded as standard items in itemset mining.

The problems of finding these frequent itemsets are fundamental in data mining, and depending on the applications, fast implementations for solving the problems are needed. In our work, we use the optimised $L C M$ algorithm [45]. LCM uses a prefix preserving closure extension to completely enumerate closed itemsets. This allows counting the support of an itemset efficiently during the mining process.

The LCM algorithm [45] supports database reduction, so that it can handle dense traditional datasets in short time and computes frequencies in linear time. It includes a strong pruning method to further reduce the computation time when the number of large frequent itemsets is small. It also generates closed itemsets with no duplication. For all these reasons, LCM is preferred over the well-known APRIORI algorithm [3]. Note though that the outcome does not depend on the choice of mining algorithm.

Encoding a new image with FLHs: Given a new image, we extract features by dense sampling and assign them to visual words. For each feature, we compute a LBOW around it, considering its $\mathrm{K}$ spatial nearest neighbours. Given this LBOW $\mathbf{x}$, we convert it into a transaction $x$ and check for each FLH pattern $t \in \Upsilon$ whether $t \subseteq x$. If $t \subseteq x$ is true, then $\mathbf{x}$ is an instance of the FLH pattern $t$. The frequency of a pattern $t$ in a given image $I_{j}$ (i.e., the number of instances of $t$ in $\left.I_{j}\right)$ is denoted as $F\left(t \mid I_{j}\right)$. We again refer to figure 1 for an example. 
3.2 Finding the best FLHs for image classification

We want to use the FLH set $\Upsilon$ as a new set of mid-level features to represent an image. To this end, we first need to select the most useful FLH patterns from $\Upsilon$ because i) the number of generated FLH patterns is huge (several millions) and ii) not all discovered FLH patterns are equally relevant for the image classification task. Usually, relevant pattern mining methods select patterns that are discriminative and not redundant. On top of that, we introduce a new selection criterion, representativity, that takes into account that, when using LBOW, a single image generates multiple transactions. As a result, some patterns may be frequent and considered discriminative but they may occur in very few images (e.g. due to repetitive structures). We believe that such features are not representative and therefore not the best choice for image classification. A good FLH pattern should be at the same time discriminative, representative and non-redundant. In this section we discuss how we select such patterns.

Relevance criterion: We use two criteria for pattern relevance: a discriminativity score $D(t)[9]$ and a new representativity score $O(t)$.

The overall relevance of a pattern $t$ is denoted by $S(t)$ defined as:

$$
S(t)=D(t) \times O(t)
$$

We claim that if a pattern $t$ has a high relevance score $S(t)$, it is likely to be discriminative and repeatable across images, hence suitable for classification.

Discriminativity score: To find discriminative patterns, we follow the entropybased approach of [9], where a discriminativity score $D(t)(0 \leq D(t) \leq 1)$ for a pattern $t$ is defined as:

$$
D(t)=1+\frac{\sum_{c} p(c \mid t) \cdot \log p(c \mid t)}{\log C}
$$

with $p(c \mid t)$ the probability of class $c$ given the pattern $t$, computed as follows:

$$
p(c \mid t)=\frac{\sum_{j=1}^{N} F\left(t \mid I_{j}\right) \cdot p\left(c \mid I_{j}\right)}{\sum_{j=1}^{N} F\left(t \mid I_{j}\right)} .
$$

Here, $I_{j}$ is the $j^{t h}$ image and $N$ is the total number of images in the dataset. $p(c \mid I)=1$ if the class label of $I_{j}$ is $c$ and 0 otherwise. A high value of $D(t)$ implies that the pattern $t$ occurs only in very few classes. Note that in Eq. 2, the term $\log C$ is used to make sure that $0 \leq D(t) \leq 1$. 
Representativity score: The second factor for the relevance $S(t)$ is the representativity $O(t)$. To compute it, we compare the distribution of the patterns over all the images with the optimal distribution with respect to a class $c$. A pattern having an optimal distribution is called an optimal pattern and denoted by $t_{c}^{*}$ for class $c$. This optimal distribution is such that i) the pattern occurs only in images of class $c$, i.e. $p\left(c \mid t_{c}^{*}\right)=1$ (giving also a discriminativity score of 1$)$, and ii) the pattern instances are equally distributed among all the images of class $c$, i.e. $\forall I_{j}, I_{k}$ in class $c, p\left(I_{j} \mid t_{c}^{*}\right)=p\left(I_{k} \mid t_{c}^{*}\right)=\left(1 / N_{c}\right)$ where $N_{c}$ is the number of images of class $c$.

To find patterns with distributions close to the optimal one, we define the representativity score of a pattern $t$ denoted by $O(t)$. It considers the divergence between the optimal distribution for class $c p\left(I \mid t_{c}^{*}\right)$ and the distribution for pattern $t p(I \mid t)$, and then takes the best match over all classes:

$$
O(t)=\max _{c}\left(\exp \left\{-\left[D_{\mathrm{KL}}\left(p\left(I \mid t_{c}^{*}\right)|| p(I \mid t)\right)\right]\right\}\right)
$$

where $D_{K L}(. \|$.$) is the Kullback-Leibler divergence between two distributions.$ The quantity $p(I \mid t)$ is computed empirically from the frequencies $F\left(t \mid I_{j}\right)$ of the pattern $\mathrm{t}$ :

$$
p(I \mid t)=\frac{F(t \mid I)}{\sum_{j} F\left(t \mid I_{j}\right)}
$$

Redundant patterns: We propose to remove redundant patterns in order to obtain a compact representative set of FLHs. We take a similar approach as in [48] to find affinity between patterns. Two patterns $t$ and $s \in \Upsilon$ are redundant if they follow similar document distributions, i.e if $p(I \mid t) \approx p(I \mid s) \approx p(I \mid\{t, s\})$ where $p(I \mid\{t, s\})$ gives the document distribution given both patterns $\{t, s\}$.

$$
p(I \mid\{t, s\})=\frac{F(t \mid I)+F(s \mid I)}{\sum_{j} F\left(t \mid I_{j}\right)+F\left(s \mid I_{j}\right)}
$$

We define the redundancy $R(s, t)$ between two patterns $s, t$ as follows:

$R(s, t)=\exp \left\{-\left[p(t) \cdot D_{K L}(p(I \mid t) \| p(I \mid\{t, s\}))+p(s) \cdot D_{K L}(p(I \mid s) \| p(I \mid\{t, s\}))\right]\right\}$

where $p(t)$ is the probability of pattern $t$ :

$$
p(t)=\frac{\sum_{I_{j}} F\left(t \mid I_{j}\right)}{\sum_{t_{j} \in \Upsilon} \sum_{I_{j}} F\left(t_{j} \mid I_{j}\right)}
$$

Note that $0 \leq R(s, t) \leq 1$ and $R(s, t)=R(t, s)$. For redundant patterns, $D_{K L}(p(I \mid t) \| p(I \mid t, s)) \approx D_{K L}(p(I \mid s) \| p(I \mid t, s)) \approx 0$ which increases the value of $R(s, t)$. 
Finding the most suitable patterns for classification: We are interested in finding the most suitable pattern subset $\chi$ where $\chi \subset \Upsilon$ for classification. To do this we define the gain of a pattern $t$ denoted by $G(t)$ s.t. $t \notin \chi$ and $t \in \Upsilon$ as follows:

$$
G(t)=S(t)-\max _{s \in \chi}\{R(s, t) \cdot \min (S(t), S(s))\}
$$

In Eq. 9, a pattern $t$ has a higher gain $G(t)$ if it has a higher relevance $S(t)$ (i.e. it is discriminative and representative) and if the pattern $t$ is non redundant with any pattern $s$ in set $\chi(i . e . R(s, t)$ is small). To find the best $k$ patterns we use the following greedy process. First we add the most relevant pattern to the relevant pattern set $\chi$. Then we search for the pattern with the highest gain (non redundant but relevant) and add this pattern into the set $\chi$ until $k$ patterns are added (or until no more relevant patterns can be found).

\subsection{Kernel function for effective pattern classification}

After computing the $k$ most relevant and non-redundant FLHs, we can represent each image using a new representation called bag-of-FLHs by counting the occurrences of such FLHs in the image. Let $L$ be such a bag-of-FLHs for the image $I_{L}$ and $M$ be the bag-of-FLHs for the image $I_{M}$. We propose to use the kernel function

$$
K(L, M)=\sum_{i} \min (\sqrt{L(i)}, \sqrt{M(i)})
$$

to find the similarities between the bag-of-FLHs of $L$ and $M$. Here $L(i)$ is the frequency of the $i^{t h}$ selected pattern in histogram $L$. This kernel provides good classification accuracies for our frequent pattern-based image representation. It is a standard histogram intersection kernel but with non-linear weighting. This reduces the importance of highly frequent patterns and is necessary since there is a large variability in pattern frequencies. Similar power-low normalization methods are used in improved Fisher Vector-based methods [33, $11]$.

\section{GRID-FLH: Incorporating global spatial information to FLH}

Finally, we propose a variant of bag-of-FLHs that incorporates both global and local spatial information. We build on the spatial pyramid idea [24] and apply it in our FLH mining framework. First we create LBOW for all features in the image. Then we discover grid-specific relevant FLH patterns by employing the process described in Section 3.2. For each image, we concatenate these grid-specific bag-of-FLH representations to create a new representation called GRID-FLH. The GRID-FLH is a more structured local-global representation with more flexibility than traditional spatial pyramids [24]. Note that we mine FLHs specific to a grid cell from all the images and then create a bag-of-FLHs 
in a grid specific way. As a result each grid-cell uses a different set of FLH patterns.

\section{Experimental Setup and Evaluations}

First, we introduce the datasets used to evaluate our $F L H$-based method in section 5.1. Then, we compare our method with the most relevant baselines in section 5.2 using some default parameters (standard SIFT descriptors with a dictionary size of 200 visual words and transactions created using 5 spatial neighbours). In section 5.3, we analyze our parameters and design choices. In section 5.4, we demonstrate on the importance of the selection of the most relevant patterns and the use of an appropriate kernel. In section 5.5 we perform some experiments to evaluate the effect of our choices (in particular of our chosen parameters) for standard BOW-based methods. In section 5.6 we evaluate the effect of our parameters on the mining step. Finally, in section 5.7 we evaluate the GRID-FLH extension.

\subsection{Datasets and evaluation criteria}

We evaluate the new bag-of-FLH (hereafter denoted by just FLH) approach on several challenging natural image datasets: GRAZ-01 [32], Oxford-Flowers17 [30], 15-Scenes [24], Land-Use [49] and the PASCAL-VOC2007 dataset [15].

The GRAZ-01 dataset consists of two object classes (bike and person) and a complex yet representative background class. For each object class (bike or person) we randomly sample 100 negative images (50 from the background class and 50 from the other object class) and 100 positive images, as done in [24]. The Oxford-Flowers dataset contains 17 flower categories where each category contains 80 images. We randomly select 60 images from each category for training and 20 images for testing as in [30]. The 15-Scenes dataset contains 15 scene categories. This dataset is useful for evaluating scene classification. From each scene category, 100 randomly selected images are used for training and the rest are used for testing as in [24]. The Pascal-VOC-2007 dataset consists of 20 object classes and 9,963 images. This dataset is one of the most interesting image classification benchmarks. The data has been split into $50 \%$ for training/validation and $50 \%$ for testing. Land-Use [49] is a new dataset consisting of 2100 images of area imagery of various urban areas. There are 21 classes including various spatial structures and homogeneous textures. For this dataset, we also keep $50 \%$ of the images for training and $50 \%$ for testing.

We use classification accuracy to evaluate our results on the Oxford-Flower and Land-Use datasets and the mean classification accuracy computed over per-class-based classification accuracies for the 15-scenes dataset as done in the literature (and for comparison purpose). For the GRAZ-01 dataset we report ROC equal error rate. For the Pascal-VOC-2007 dataset, we report the mean average precision or mAP. Because of the size of the Pascal-VOC2007 and Land-Use datasets, we did not perform any baseline comparisons nor 
parameter optimizations for them, we simply report the results obtained with the parameters optimized for the other datasets.

For the initial baseline experiments (and for all datasets), we start from SIFT descriptors [29] densely sampled over the image with patches of size $16 \times 16$ pixels and a grid spacing of 8 pixels.

We use the K-means algorithm to create the visual dictionaries and LIBSVM $[7]^{1}$ to train an SVM. We use the square root intersection kernel for FLH-based methods as presented in Section 3.3.

\subsection{Initial comparison with baseline methods}

We compare our FLH-based method using some default settings (spatial neighborhood size $\mathrm{K}=5$, standard SIFT, dictionary size of 200) with BOW-based image classification, spatial pyramid matching (SPM) [24], visual word-based standard frequent itemset mining with binarized local bag-of-words (denoted by $F I M)$ and with the $B 2 S$ [23] representation. For all baseline methods, we use the same LCM algorithm for pattern mining and an intersection kernel for SVM. We use a maximum of 10.000 patterns for all mining methods. We also report results for the mining-based method combined with $B O W$ using an average Kernel (denoted by BOW+Mining-Method). The results are shown in Table 1.

Table 1: Comparison with baseline methods. Classification accuracies are reported for GRAZ-01, Oxford-Flower17 and 15-Scenes datasets with a local neighborhood size of $\mathrm{K}=5$.

\begin{tabular}{|l|l|l|l||c|c|c||c|c|c|}
\hline & Dict. & \multicolumn{2}{|c|}{ Baselines } & \multicolumn{3}{c||}{ Mining Methods } & \multicolumn{3}{c|}{ Mining+BOW } \\
\hline & size & BOW & SPM & FIM & B2S & FLH & FIM & B2S & FLH \\
\hline GRAZ-Person & 200 & 79.4 & 79.7 & 80.5 & 81.8 & 83.5 & 81.8 & 83.4 & $\mathbf{8 4 . 0}$ \\
\hline GRAZ-Bike & 200 & 76.8 & 79.6 & 78.0 & 78.4 & 81.3 & 80.6 & 81.8 & $\mathbf{8 2 . 5}$ \\
\hline Flower & 200 & 56.4 & 57.3 & 54.7 & 55.3 & 59.0 & 60.8 & 64.4 & $\mathbf{7 1 . 1}$ \\
\hline 15-Scenes & 200 & 73.6 & $\mathbf{8 1 . 0}$ & 67.9 & 69.6 & 70.5 & 73.3 & 74.1 & 76.5 \\
\hline
\end{tabular}

FIM is comparable with SPM (except for 15-Scenes) while B2S (which is an alternative lossless histogram transformation approach as explained in Section 2) slightly outperforms FIM. The FLH-based method outperforms all the baseline methods as well as all the other mining-based methods, except in the 15-Scenes dataset. This result shows the importance of not losing information during the transaction creation time. We believe that our FLH-based method improves the results over B2S because it does not generate artificial visual patterns (i.e. patterns not actually present in the data set) while B2S does. The combination of $B O W s$ and bag-of-FLHs gives better results compared to all other methods and is an indication of the complementary nature of both

1 http://www.csie.ntu.edu.tw/ cjlin/libsvm/ 
Table 2: The effect of dictionary size on FLH-based methods using SIFT-128. Classification accuracy on training data using cross-validation.

\begin{tabular}{|c|c|c|c|c|c|c|c|c|}
\hline & \multicolumn{2}{|c|}{ Oxford-Flower } & \multicolumn{2}{|c|}{ 15-Scenes } & \multicolumn{2}{c|}{ GRAZ-Bike } & \multicolumn{2}{c|}{ GRAZ-Person } \\
\hline Dict. Size & FLH & FLH + BOW & FLH & FLH + BOW & FLH & FLH + BOW & FLH & FLH + BOW \\
\hline 800 & $\mathbf{6 4 . 4}$ & 64.8 & $\mathbf{7 1 . 8}$ & 75.4 & $\mathbf{8 4 . 4}$ & 83.5 & 82.3 & 83.4 \\
\hline 400 & 57.1 & 65.6 & 71.2 & 76.2 & 84.2 & $\mathbf{8 5 . 9}$ & 83.3 & 83.9 \\
\hline 200 & 56.2 & 68.9 & 70.5 & 76.5 & 81.3 & 85.2 & $\mathbf{8 3 . 5}$ & $\mathbf{8 4 . 0}$ \\
\hline 100 & 54.7 & $\mathbf{7 0 . 3}$ & 65.8 & $\mathbf{7 6 . 9}$ & 80.5 & 84.3 & 83.1 & 81.9 \\
\hline 50 & 50.7 & 56.2 & 60.1 & 75.1 & 80.3 & 85.0 & 81.2 & 82.3 \\
\hline
\end{tabular}

representations. Especially the improvement for the Flowers dataset is remarkable. FLH outperforms other baselines for GRAZ-01 dataset as well. For the 15-Scenes dataset (with the default parameters) spatial pyramids and even just BOW outperform the other methods. These initial experiments, without parameter optimization, already clearly hint at the power of our new image representation.

\subsection{Parameter selection and optimization}

In this set of experiments we analyze the effect of several parameters of our method: dictionary size, SIFT feature size, and local neighborhood size. We use a three-fold cross-validation on training data to optimize our parameters using Oxford-Flower, 15-Scenes and GRAZ-01 datasets.

In the remaining experiments (sections 5.4, 5.5, 5.6 and 6), we then use the found optimal parameters to test our FLH-based methods on the test sets.

Dictionary size: We are interested in exploiting local spatial information using mining methods. Larger visual dictionaries may restrict the possibility of exploiting co-occurring local statistics. To evaluate this phenomenon we evaluate the effect of the dictionary size on our FLH-based method. We report results for $F L H$ and $F L H+B O W$ with different dictionary sizes (Table 2). Note that when combining $F L H$ and $B O W$ we do not reduce the dictionary size for $B O W$, but always use a dictionary size of 800 , as large dictionaries have proven to perform better for BOW. Results decrease when the dictionary size is reduced. However, the results improve with reduced dictionaries for $F L H+B O W$, up to some level. This indicates that with a smaller dictionary size (up to some level) FLHs complementarity with $B O W$ representation increases.

Smaller dictionaries reduce the discriminativity of the visual primitives. However, this does not seem to affect the discriminativity of the FLH patterns, which may even become more robust and stable when combined with BOW. Therefore, smaller dictionaries created using even less discriminative features might be better suited for $F L H$. This is tested in the next experiment. 


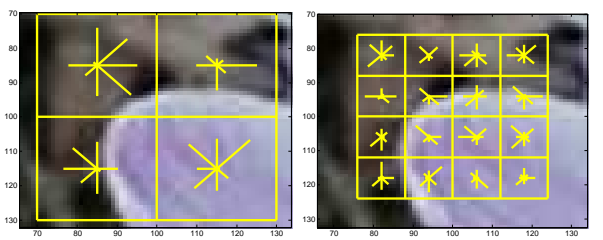

Fig. 3: Spatial bining of SIFT32 (left) vs SIFT128 (right)

Table 3: Effect of SIFT-32 features on FLH. Classification accuracy on training data using cross-validation.

\begin{tabular}{|c|c|c|c|c|c|c|c|c|}
\hline & \multicolumn{2}{|c|}{ Oxford-Flower } & \multicolumn{2}{|c|}{ 15-Scenes } & \multicolumn{2}{c|}{ GRAZ-Bike } & \multicolumn{2}{c|}{ GRAZ-Person } \\
\hline Dict. Size & FLH & FLH + BOW & FLH & FLH + BOW & FLH & FLH + BOW & FLH & FLH + BOW \\
\hline 800 & 69.1 & 79.4 & $\mathbf{7 0 . 4}$ & 74.9 & 83.9 & 85.1 & 83.5 & 84.1 \\
\hline 400 & 69.5 & 80.1 & 70.0 & 75.6 & 84.8 & 85.8 & 83.6 & 84.2 \\
\hline 200 & 70.4 & 80.6 & 68.9 & $\mathbf{7 5 . 8}$ & 84.8 & 87.2 & 83.6 & 84.4 \\
\hline 100 & $\mathbf{7 2 . 7}$ & $\mathbf{8 0 . 7}$ & 65.4 & 75.3 & 85.4 & $\mathbf{8 7 . 2}$ & $\mathbf{8 3 . 7}$ & 84.4 \\
\hline 50 & 70.0 & 80.4 & 61.0 & 75.0 & $\mathbf{8 6 . 1}$ & 86.7 & 82.0 & $\mathbf{8 5 . 1}$ \\
\hline
\end{tabular}

Less discriminative features: We evaluate the effect of less discriminative features on FLH using the same four datasets and local neighborhood size of 5 (Table 3). For this we use SIFT32 features that are extracted like the standard SIFT (referred to as SIFT128) but performing spatial binning of $(2 \times 2)$ instead of $(4 \times 4)$ (See Fig.3). Results are shown in Table 3, for FLH with SIFT32 by itself, as well as when combined with $B O W$ (using SIFT128 and a 800 dimensional vocabulary for the BOW). For most of the settings, we obtain better results on all datasets except 15-Scenes, when compared to the case of SIFT-128.

Larger local neighborhoods: The use of smaller dictionaries and less discriminative SIFT features allows the $F L H$-based method to exploit larger neighborhoods. To evaluate this relation, we run some further experiments on the same datasets - see Fig. 4 . The best results are obtained when reducing both SIFT feature size and dictionary size while increasing the neighborhood size of the local spatial histograms. For Oxford-flower, the best classification accuracy obtained with SIFT features is $91.0 \%$ for a dictionary size of 100 words, SIFT32 features and a neighborhood size of 25. A similar trend is observed for 15-Scenes dataset and the GRAZ dataset. Note that this is a larger neighborhood size (covering up to $48 \times 48$ pixels) than what is typically used in the literature $[53,20]$.

We can conclude that the $F L H$-based method obtains its best results when exploiting larger patterns with smaller dictionaries and less discriminative primitive features.

From now on, for all $F L H$-based experiments we use the SIFT32 descriptor and a neighborhood size of 25 neighbors. On average best performance is 


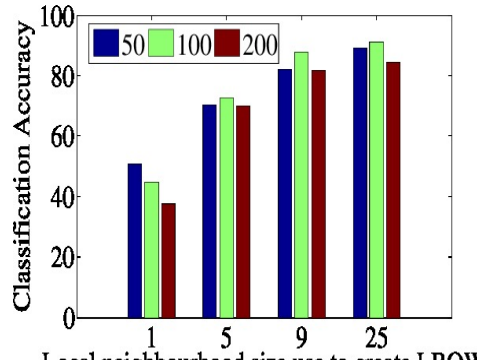

Local neighbourhood size use to cre

(a) Oxford-Flower

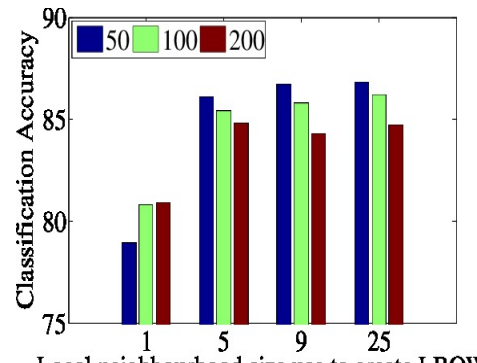

(c) GRAZ-Bike

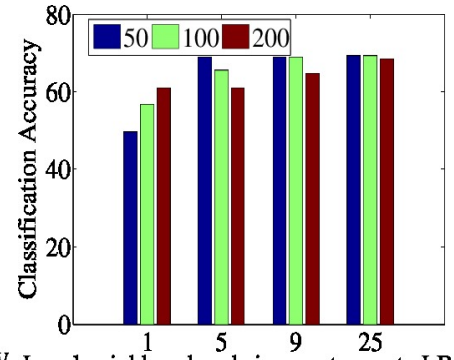

(b) 15-Scenes

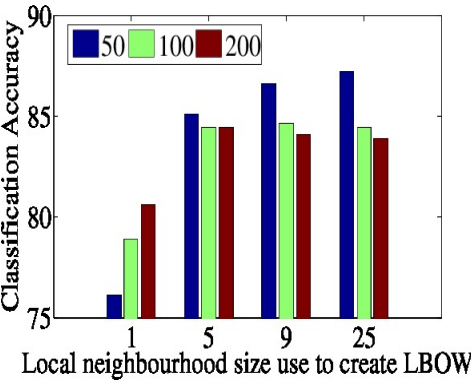

(d) GRAZ-Person

Fig. 4: Effect of neighborhood size (K, horizontal axis), dictionary size (color coded) and SIFT-32 on classification accuracy for the (a) Oxford-Flower (b)15-Scenes (c) GRAZ-Bike (d) GRAZ-Person on training data using crossvalidation.

obtained around 100 visual words or less for most of the datasets. As a result hereafter we use a visual dictionary size of 100 words.

\subsection{Effect of relevant pattern mining and of the kernel functions}

FLH mining algorithms can generate a large number of FLH patterns (in our case, 1-20 million) before the relevant pattern mining step. Therefore, we select the most relevant-non-redundant ones, as described in Section 3.2. Here we evaluate the importance of this pattern selection step by comparing different criteria: the most frequent (Frq.), the most representative-nonredundant (Rps.)(eq. 4), the most discriminative-non-redundant (Disc.)(eq.2) and the most relevant-non-redundant (Rel.) (i.e. representative, discriminative and non-redundant) patterns (see Table 4). We always select the top 10.000 patterns for each criterion which we believe is sufficient to obtain good results. These results clearly show that only selecting the top-k most frequent patterns (as often done in computer vision literature, e.g. [31]) is not a good choice for classification. Both representativity and discriminativity criteria alone also do 

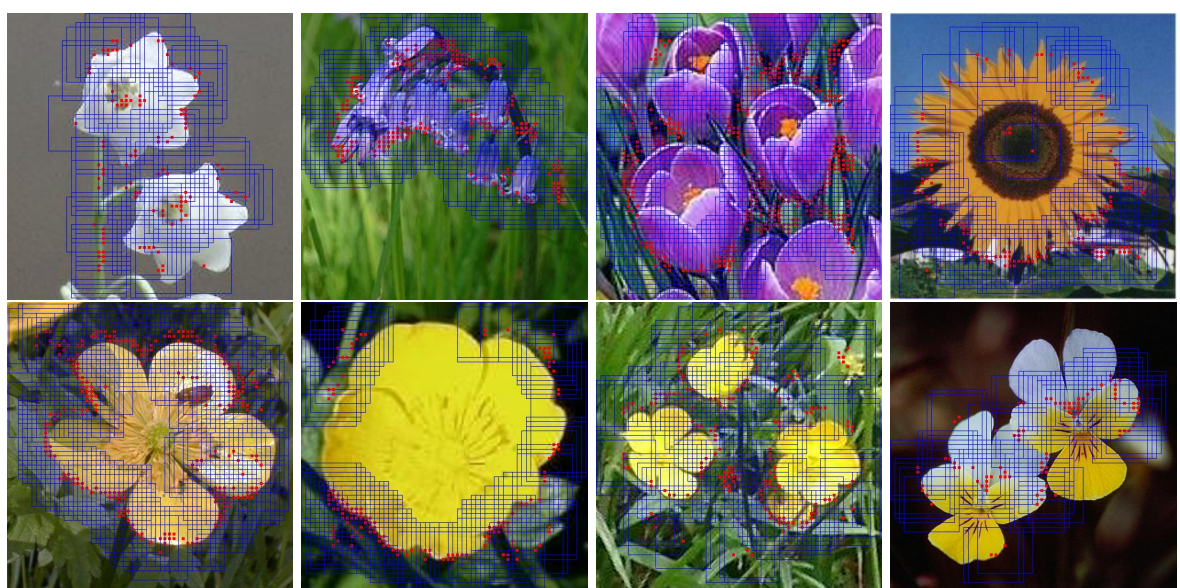

Fig. 5: FLH patterns using SIFT32 where each red dot represents the central location and the blue square represents the size of the FLH pattern. Each pattern is generated from a $48 \times 48$ pixel image patch. In this region there are 25 features each covering a $16 \times 16$ pixel patch and overlapping $50 \%$ with their neighbors. Note how the relevant non-redundant patterns seem to capture most of the relevant shape information in the images.

not provide the best results. It's the relevant non-redundant FLH patterns that are the most suitable for classification.

Some of these relevant and non-redundant FLH patterns are shown in Fig. 5. These selected most relevant FLH patterns seem to capture most of the relevant shape information in an image. In Fig. 6 we show the most relevant pattern for seven different flower categories. Note how each relevant pattern captures several local feature configurations. All these configurations seem visually similar, even though they show quite some variability as well, especially when compared to the mid-level features obtained by methods that start from a rigid HoG-based representation.

As can be seen from Fig. 5, these FLH patterns cover relatively large regions. Fig. 7 shows the distribution of pattern size. Most of the pattern sizes are between length 5 to 8 . This implies that most of the local histogram patterns consist of 5-8 non-zero components. Very large patterns and very small patterns have very low probability. The relevant-non redundant patterns contain slightly less items.

In Table 5, we evaluate the effect of the square root intersection kernel using relevant non-redundant FLH patterns on Oxford-Flower, GRAZ-01 and 15-Scenes datasets. The proposed square-root weighting increases the classification accuracy for all datasets, both when using the linear kernel and the non-linear intersection kernel.

The square-root intersection kernel combines an intersection kernel and a power kernel with power 0.5. Both kernels decrease the impact of very fre- 


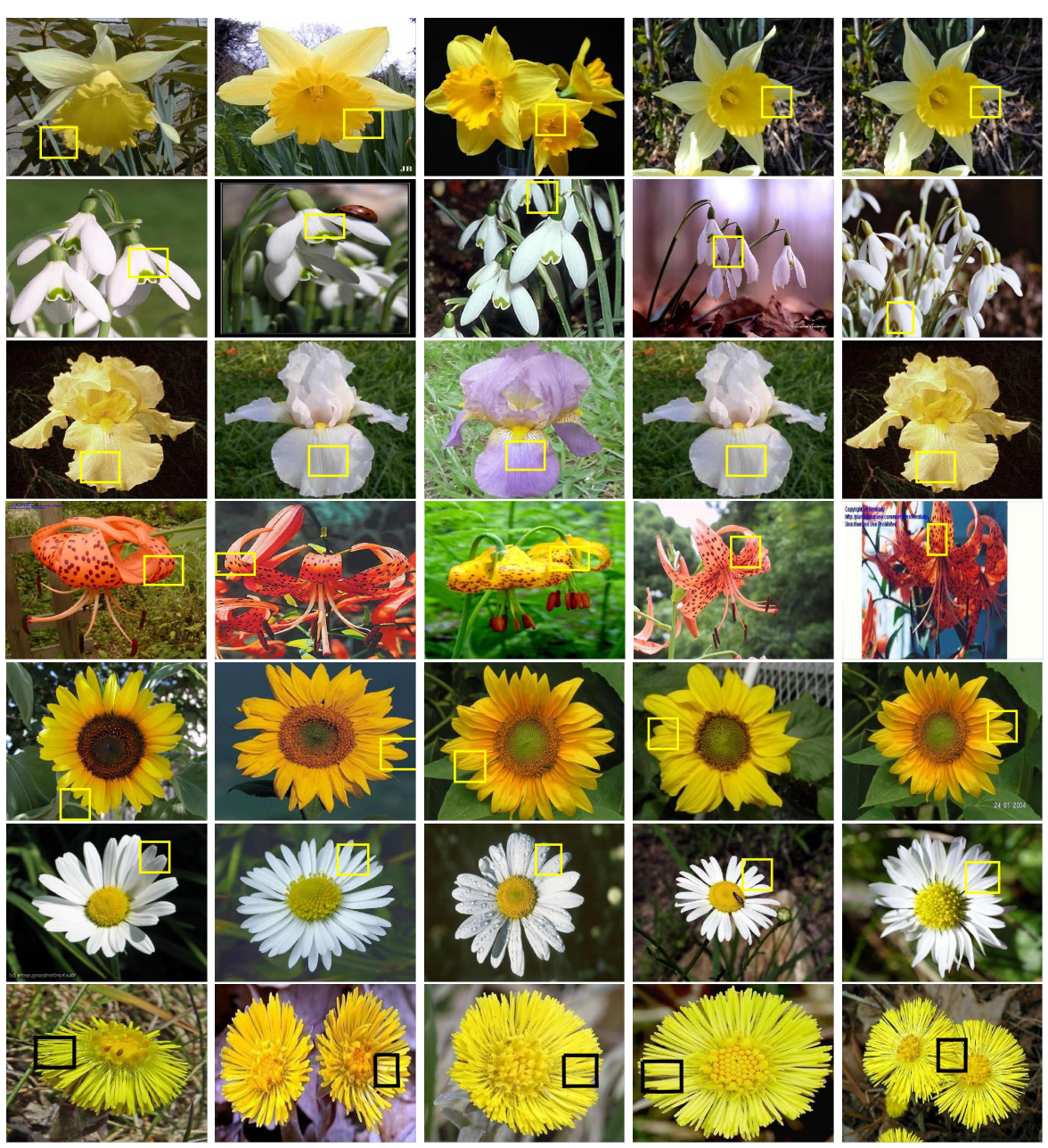

Fig. 6: Each row represents the most relevant FLH pattern for a given flower category. Note the flexibility of our representation. While it's always the same FLH pattern, the appearance of the corresponding patches varies a lot.

quent patterns. As shown by experiments, this kernel is better than either kernel separately (see Table 5). This shows that the intersection kernel and power kernel with power 0.5 do not reduce sufficiently the influence of frequent patterns. An alternative would be to use a smaller power than 0.5, (e.g. 0.25). The main advantage of this is that the power kernel can be implemented as a simple normalization of the features. Efficient linear classifiers can be used subsequently as opposed to costly non-linear classifiers. We plan to investigate this effect in our future work. 
Table 4: Effect of relevant pattern mining on classification performance using FLH

\begin{tabular}{|l|l|l|l|l|}
\hline Criterion & Frq. & Rps. & Disc. & Rel. \\
\hline Flower & 65.6 & 84.2 & 90.9 & 92.5 \\
\hline 15-Scenes & 66.8 & 69.6 & 67.4 & 70.4 \\
\hline GRAZ-01 & 82.9 & 89.8 & 90.2 & 91.5 \\
\hline
\end{tabular}

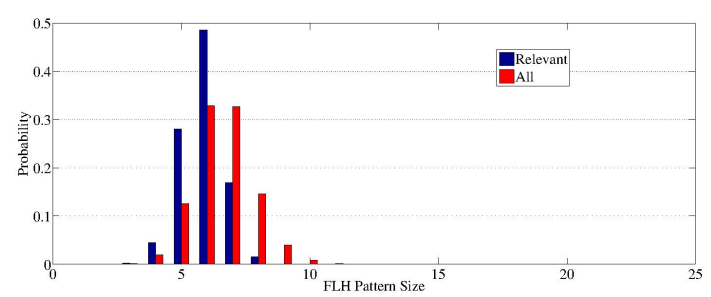

Fig. 7: The distribution of the pattern size

Table 5: Effect of the kernel on classification performance using FLH

\begin{tabular}{|l|l|l|l|l|}
\hline$K(\mathbf{x}, \mathbf{y})=$ & $\mathbf{x} \cdot \mathbf{y}^{t}$ & $\sqrt{\mathbf{x}} \cdot \sqrt{\mathbf{y}}^{t}$ & $\sum_{i} \min \left(\mathbf{x}_{\mathbf{i}}, \mathbf{y}_{\mathbf{i}}\right)$ & $\sum_{i} \min \left(\sqrt{\mathbf{x}_{\mathbf{i}}}, \sqrt{\mathbf{y}_{\mathbf{i}}}\right)$ \\
\hline Flower & 89.5 & 92.0 & 91.2 & $\mathbf{9 2 . 5}$ \\
\hline 15-Scenes & 68.0 & 68.9 & 69.0 & $\mathbf{7 0 . 4}$ \\
\hline GRAZ-01 & 88.5 & 89.5 & 89.8 & $\mathbf{9 1 . 5}$ \\
\hline
\end{tabular}

Table 6: Effect of larger patches $(48 \times 48)$ and dictionary size on BOW/SPM based image classification.

\begin{tabular}{|c|c|c|c|c|c|c|c|c|}
\hline & \multicolumn{2}{|c|}{ Oxford-Flower } & \multicolumn{2}{c|}{ 15-Scenes } & \multicolumn{2}{c|}{ GRAZ-Bike } & \multicolumn{2}{c|}{ GRAZ-Person } \\
\hline Dict. Size & BOW & SPM & BOW & SPM & BOW & SPM & BOW & SPM \\
\hline 100 & 55.0 & 60.8 & 66.5 & 73.7 & 79.5 & 83.0 & 79.5 & 80.0 \\
\hline 1000 & 62.9 & 66.5 & 72.1 & 76.4 & 80.0 & 80.0 & 83.0 & 83.0 \\
\hline 4000 & 65.5 & 67.4 & 72.9 & 76.2 & 79.0 & 80.5 & 83.0 & 82.5 \\
\hline
\end{tabular}

5.5 Effect of larger patches and dictionary size on BOW based image classification

One might argue that the FLH-based method with the optimized parameters described in the previous section outperforms the BOW baseline because of the use of larger patches and much simpler features. To evaluate this hypothesis, we perform another experiment using BOW with SIFT-32 descriptors extracted from large patches of size $48 \times 48$ pixels. By varying the dictionary size we evaluate the performance of the BOW and SPM methods. The results are shown in Table 6 . They show that just increasing the patch size of SIFT descriptors is not sufficient to increase the classification accuracy for BOW-based methods. For example for Oxford-Flower dataset the best performance using SPM is $67.4 \%$ while FLH reported $92.5 \%$. For 15 -Scenes dataset $F L H+B O W$ reported 83.0 while the best for SPM is $76.4 \%$. 
5.6 Effect of larger spatial neighborhoods and smaller dictionary size on frequent pattern based image classification

To evaluate the effect of larger spatial neighborhoods and smaller visual dictionaries on all pattern-based methods (i.e. FIM, B2S and FLH) we perform another experiment using SIFT-32 descriptors. Transactions are created using a spatial neighborhood size of 25 neighbors. Results are shown in Fig. 8. We report results using both histogram intersection kernel (Ints.) and square root histogram intersection kernel (Sqrt. Ints.). For all three methods (FIM, B2S and FLH), the traditional top-k most frequent pattern selection method performs poorly. All methods benefit from the relevant pattern selection but the FLH-based method benefits the most (the top-k most frequent pattern results are improved by $27 \%$ while it is $19 \%$ for B2S and $17 \%$ for FIM for Oxford-Flower). A similar trend can be observed for the other datasets too. It is clear from this experiment that smaller visual dictionaries that are constructed from less discriminative SIFT are only helpful if we use the relevant pattern selection step. Since the binary histogram transformation used in the FIM method loses some valuable information, this method does not benefit that much from the relevant pattern selection step nor from the reduction of the dictionary size, SIFT dimension and the increase of the spatial neighborhood. We believe that the significant 10\% improvement (on Oxford-Flower) of FLH over B2S when using the same parameters is due to two reasons; (1) FLH local representation is more expressive and explicit compared to B2S, (2) FLH does not generate artificial patterns (ghost patterns).

\subsection{Effect of GRID-FLH vs Spatial pyramids}

In this section we compare the extension of bag-of-FLHs called GRID-FLH introduced in section 4. We compare GRID-FLH with FLH and spatial pyramids [24] with BOW (SPM-BOW) and spatial pyramids with FLH (SPMFLH). SPM-BOW is the standard spatial pyramids applied over BOW while in SPM-FLH spatial pyramids are computed over bag-of-FLHs. Results are reported in Table 7. GRID-FLH consistently improves over FLH and spatial pyramid variants (both SPM-BOW and SPM-FLH). Especially in scene classification the improvement obtained by GRID-FLH is significant.

Table 7: Effect of GRID-FLH vs Spatial pyramids

\begin{tabular}{|l|l|l|l|l|}
\hline Method & FLH & SPM-BOW & SPM-FLH & GRID-FLH \\
\hline Flower & 92.5 & 57.3 & 92.6 & $\mathbf{9 2 . 9}$ \\
\hline 15-Scenes & 70.4 & 81.0 & 82.8 & $\mathbf{8 6 . 2}$ \\
\hline GRAZ-01 & 91.6 & 84.3 & 92.4 & $\mathbf{9 3 . 8}$ \\
\hline
\end{tabular}




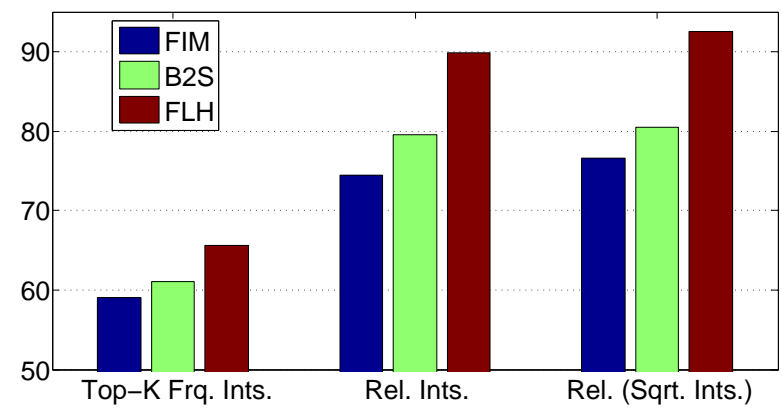

(a) Oxford-Flower

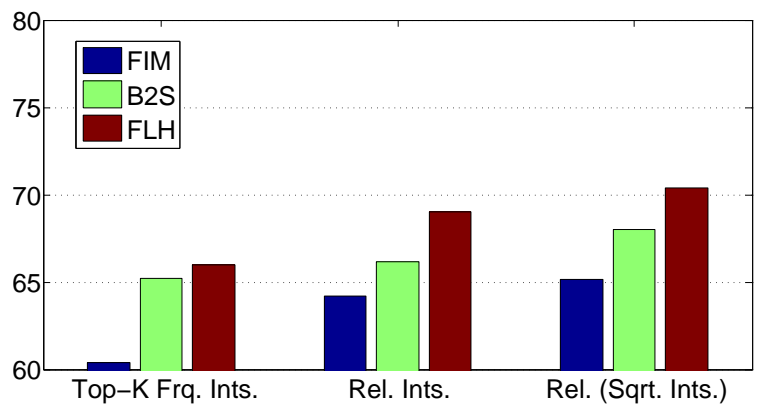

(b) 15 -Scenes

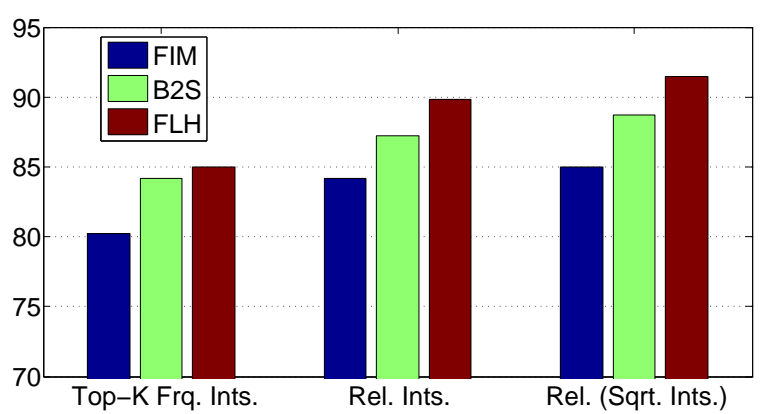

(c) GRAZ-01

Fig. 8: Effect of larger spatial neighborhoods (25), smaller dictionary size (100) and SIFT-32 descriptor on frequent pattern-based image classification.

\section{Comparison to state-of-the-art methods}

6.1 Comparison with non-mining methods

In this section we compare FLH with non-mining methods that exploit local structural statistics. Specifically we compare our method with the spatial pyramid co-occurrence method (SPCK) of Yang et al. in [49] and the unbounded- 
order spatial features method of Chen and Zhang [51]. We also compare our method with the mid-level feature construction method of Boureau et al. in [6] and the PDK method [27] which uses proximity distribution kernels based on geometric context for category recognition. Results are reported in Table 8 . GRID-FLH outperforms all other non-mining methods for both GRAZ-01 and 15-Scenes datasets. Note that for these methods, no results were reported for the Oxford-flower dataset.

The mid-level features method of [6] using macro-features seems to work quite well on 15-Scenes. This method also uses dense SIFT features but a visual dictionary of 2048. In this method the sparsity and supervision is enforced during the feature construction. The Bag-of-FLH representation, on the other hand, is quite sparse after the relevant pattern mining step. For example, in the case of Oxford-Flower dataset, there were $17.6 \%$ non-zero bins before the relevant pattern mining step and $5.13 \%$ non-zeros bins after. One of the key differences between the macro-features and FLH is that macro-features capture very small neighborhood of $2 \times 2$ while FLHs capture comparatively larger neighborhoods of $5 \times 5$. Secondly for macro-features larger discriminative and supervised dictionaries seem to work well. For an unsupervised smaller dictionary of size 1048 macro-features reported only $83.6 \%$.

Neither the spatial pyramid co-occurrence method of Yang et al. in [49] nor the unbounded-order spatial features method of Chen and Zhang [51] work as good as our GRID-FLH method. This could be due to the fact that none of these methods capture database wide spatial statistics.

Table 8: Comparison with non-mining methods

\begin{tabular}{|l|l|l|l|}
\hline Dataset & GRAZ-Bike & GRAZ-Person & 15-Scenes \\
\hline GRID-FLH & $\mathbf{9 5 . 8}$ & $\mathbf{9 1 . 4}$ & $\mathbf{8 6 . 2}$ \\
\hline FLH+BOW & 95.0 & 90.1 & 83.0 \\
\hline FLH & 94.0 & 89.2 & 70.4 \\
\hline \hline SPCK [49] & 91.0 & 87.2 & 82.5 \\
\hline PDK [27] & 95.0 & 88.0 & - \\
\hline Higher Order Features [51] & 94.0 & 84.0 & - \\
\hline Mid-Level Features [6] & - & - & 85.6 \\
\hline
\end{tabular}

\subsection{Comparison with state-of-the-art methods}

In this section we compare FLH using the parameters optimized as above with, to the best of our knowledge, the best results reported in the literature.

GRAZ-01: The results reported in Table 9 show that on average all FLHbased methods outperform the state-of-the-art. The GRID - FLH representation, combining local and global spatial information, yields the best results. 
For the "Bike" class, the higher order features [51] seem the best. But on average FLH outperforms the higher order features [51] and the co-occurrence spatial features [49].

Table 9: Equal Error Rate (over 20 runs) for categorization on GRAZ-01 dataset

\begin{tabular}{|l|c|c|c|}
\hline Method & Person & Bike & Average \\
\hline$S P C K_{+}[49]$ & 87.2 & 91.0 & 89.1 \\
\hline NBNN [4] & 87.0 & 90.0 & 88.5 \\
\hline Higher Order Features [51] & 84.0 & $\mathbf{9 4 . 0}$ & 89.0 \\
\hline FLH & 94.0 & 89.2 & 91.6 \\
\hline FLH + BOW & 95.0 & 90.1 & 92.6 \\
\hline GRID-FLH & $\mathbf{9 5 . 8}$ & 91.4 & $\mathbf{9 3 . 8}$ \\
\hline
\end{tabular}

Oxford-Flower: The results are reported in Table 10. Note that only using SIFT features we get a classification accuracy of $\mathbf{9 2 . 9 \%}$, reaching the stateof-the-art. GRID-FLH only gives an insignificant improvement of $0.4 \%$ compared to FLH. Note that we use only SIFT features for classification. Most of the other works such as $[30,38,47,17]$ use multiple features such as Hue and ColorName descriptors [46]. To the best of our knowledge the best results on Oxford-Flower17 using a single feature is reported by Rematas et al. [36], $85.3 \%$. We should mention that when we combine SIFT with color information (using the ColorName descriptor [46]) we obtain a classification accuracy of $\mathbf{9 4 . 0 \%}$ outperforming the state-of-the-art.

Table 10: Classification accuracy (over 20 runs) on the Flower dataset

\begin{tabular}{|l|c|}
\hline Method & Accuracy \\
\hline Nilsback [30] & 88.3 \\
\hline CA [38] & 89.0 \\
\hline$L_{1}-B R D[47]$ & 89.0 \\
\hline LRFF [17] & $\mathbf{9 3 . 0}$ \\
\hline Pooled NBNN Kernel [36] & 85.3 \\
\hline FLH & 92.5 \\
\hline FLH + BOW & 92.7 \\
\hline GRID-FLH & 92.9 \\
\hline
\end{tabular}

15-Scenes: Results are shown in Table 11. This dataset is strongly aligned. $F L H$ does not exploit this and therefore by itself cannot obtain state-of-theart results. However, the GRID-FLH method described in section 4 does take the global spatial information into account and achieves close to state-of-theart results $(86.2 \%)$. This is only outperformed by [54] who report $87.8 \%$ using CENTRIST and SIFT features along with LLC coding. In our defense, our 
method uses only SIFT features. As far as we know the previous best classification accuracy using SIFT features was reported by Tuytelaars et al. in [44] combining a NBNN kernel and a SPM method.

Table 11: Results on 15-Scenes dataset

\begin{tabular}{|l|c|}
\hline Method & Accuracy \\
\hline SPM & 80.9 \\
\hline SPCK $++[49]$ & 82.5 \\
\hline NBNN Kernel+SPM [44] & 85.0 \\
\hline (AND/OR) [54] & $\mathbf{8 7 . 8}$ \\
\hline FLH & 70.4 \\
\hline FLH+BOW & 83.0 \\
\hline GRID-FLH & 86.2 \\
\hline
\end{tabular}

Land-Use: Yang and Newsam proposed a spatial pyramid co-occurrence method called SPCK [49] to classify Land-Use images. Most of these images are texture dominant. They use two types of spatial predicates: the proximity predicate and the orientation predicate, to define the SPCK method. We obtain a best result of $\mathbf{7 9 . 2 \%}$ for this dataset, again outperforming best results reported in [49]. The results for Land-Use dataset are shown in Table 12.

Table 12: Results on recent Land-Use dataset

\begin{tabular}{|l|c|}
\hline Method & Accuracy \\
\hline BOW & 71.9 \\
\hline SPM & 74.0 \\
\hline$S P C K_{S P 1}[49]$ & 72.6 \\
\hline$S P C K_{S P 3}+[49]$ & 76.1 \\
\hline FLH & 76.8 \\
\hline FLH+BOW & 77.2 \\
\hline GRID-FLH & $\mathbf{7 9 . 2}$ \\
\hline
\end{tabular}

Pascal-VOC200\%: Results are reported in Table 13. For this dataset Fisher Vector $[33,8]$ is the best performing method so far and the authors report a $\mathrm{mAP}$ of 61.7 . The FLH-based method alone gives a mAP of 60.4. In combination with BOW of SIFT-128 and 5000 visual word vocabulary (with a weighted average kernel with weights learned using train/validation set), we obtain a state-of-the-art mAP of 62.8 . Note that the score for each individual class often varies a lot between the FLH+BOW and the Fisher Vector [33] method. Our method does especially well on what are known to be 'hard' classes such as bottle $(+34 \%$ improvement), dining table $(+11 \%)$, potted plant $(+16 \%)$, or tv monitor $(+23 \%)$. This suggests that both methods are complementary. To evaluate this claim we also performed another experiment in which we average the output score of Fisher vector method $[33,8]$ with the output scores 
of (FLH+BOW) method. This yields a mean average precision of $\mathbf{7 2 . 2}$. This approach clearly outperforms the state-of-the art by a significant margin. Not only this confirms the complementary nature of FLH and Fisher vectors but the improvement is consistent over every PASCAL-VOC class.

Table 13: Results on PASCAL-VOC 2007 (Mean average precision)

\begin{tabular}{|c|c|c|c|c|c|c|c|c|c|c|c|}
\hline Class & $\lambda$ & రొత్ర & 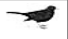 & 象 & d & 且 & $\Leftrightarrow$ & D) & $=$ & 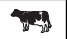 & \\
\hline Fisher Vectors (FV) & 78.8 & 67.4 & 51.9 & 70.9 & 30.8 & 72.2 & 79.9 & 61.4 & 56.0 & 49.6 & \\
\hline FLH & 67.9 & 70.6 & 41.0 & 54.6 & 64.9 & 60.9 & 85.8 & 56.6 & 59.6 & 40.0 & \\
\hline $\mathrm{FLH}+\mathrm{BOW}$ & 69.2 & 73.0 & 42.7 & 56.3 & 64.9 & 60.9 & 86.6 & 58.9 & 63.3 & 41.8 & \\
\hline $\mathrm{FLH}+\mathrm{FV}$ & 78.6 & 76.3 & 55.7 & 75.0 & 74.9 & 75.6 & 87.4 & 66.2 & 65.7 & 50.6 & \\
\hline $\mathrm{FLH}+\mathrm{BO} W+\mathrm{FV}$ & 80.0 & 78.0 & 55.9 & 76.2 & 75.5 & 75.6 & 88.1 & 67.0 & 67.3 & 51.8 & \\
\hline Class & 雨 & $\boldsymbol{t}$ & ThS & 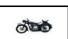 & is & 維 & $m$ & $=$ & 盆 & 무 & m.AP \\
\hline Fisher Vectors (FV) & 58.4 & 44.8 & 78.8 & 70.8 & 85.0 & 31.7 & 51.0 & 56.4 & 80.2 & 57.5 & 61.7 \\
\hline FLH & 64.7 & 47.3 & 56.6 & 65.7 & 80.7 & 46.3 & 41.8 & 54.6 & 71.0 & 77.6 & 60.4 \\
\hline $\mathrm{FLH}+\mathrm{BOW}$ & 74.3 & 48.4 & 61.8 & 68.4 & 81.2 & 48.5 & 41.8 & 60.4 & 72.1 & 80.8 & 62.8 \\
\hline FLH+FV & 75.7 & 52.4 & 78.7 & 77.0 & 88.8 & 53.9 & 51.7 & 68.7 & 83.6 & 82.0 & 70.9 \\
\hline $\mathrm{FLH}+\mathrm{BOW}+\mathrm{FV}$ & 80.9 & 51.9 & 78.9 & 77.3 & 89.8 & 58.5 & 51.8 & 72.0 & 83.9 & 84.5 & 72.2 \\
\hline
\end{tabular}

\section{Conclusion}

In this paper we show an effective method for using itemset mining to discover a new set of mid-level features called Frequent Local Histograms. Extensive experiments have proved that the proposed bag-of-FLH representation, the proposed relevant pattern mining method and the chosen kernel all improve the classification results on various datasets. We have also experimentally shown that the best results for FLH methods are obtained when exploiting a large local neighborhood with a small visual vocabulary and less discriminative descriptors. Finally, we have shown that extending a local approach such as FLH to exploit global spatial information allows us to obtain state-of-the-art results on many datasets. The experiments have also put in light the complementary nature of FLH and Fisher vectors, the combination of which can significantly increase the classification accuracies on difficult classification problems.

FLH uses dense sampling only at a single scale. This is a limitation of our approach even though we were able to obtain good results on many datasets with a single scale. Note that moving to interest points instead of densely sampled points would not resolve this issue.

As future work we propose to investigate how to push the relevant and non redundant constraints directly into the local histogram mining process to make it particularly efficient. Besides, as itemset mining is performed on the local histograms, the spatial information that can be captured in the patterns may be limited in some applications. Using graph mining methods on the grid of visual words could be an interesting alternative method to the one proposed in this paper to build bigger mid-level features. 
We also plan to further investigate pattern mining methods in image classification, image/video retrieval and multiple query image retrieval settings. Integration of Fisher Vectors into our framework may lead to further performance gains. Furthermore, we would like to further explore unsupervised relevant pattern mining techniques and how to extend FLH using Gaussian mixture-based visual word representations and probabilistic mining.

Acknowledgements The authors acknowledge the support of the iMinds Impact project Beeldcanon, the FP7 ERC Starting Grant 240530 COGNIMUND and PASCAL 2 Network of Excellence.

\section{References}

1. Agarwal, A., Triggs, B.: Multilevel image coding with hyperfeatures. Int. J. Comput. Vision 78, 15-27 (2008). DOI 10.1007/s11263-007-0072-x. URL http://dl.acm.org/citation.cfm?id=1349659.1349668

2. Agrawal, R., Imieliński, T., Swami, A.: Mining association rules between sets of items in large databases. SIGMOD Rec. pp. 207-216 (1993)

3. Agrawal, R., Srikant, R.: Fast algorithms for mining association rules in large databases. In: VLDB, pp. 487-499 (1994). URL http://portal.acm.org/citation.cfm?id=645920.672836

4. Boiman, O., Shechtman, E., Irani, M.: In defense of nearest-neighbor based image classification. In: CVPR (2008)

5. Bourdev, L., Malik, J.: Poselets: Body part detectors trained using 3d human pose annotations. In: International Conference on Computer Vision (ICCV) (2009). URL http://www.eecs.berkeley.edu/ lbourdev/poselets

6. Boureau, Y.L., Bach, F., LeCun, Y., Ponce, J.: Learning mid-level features for recognition. In: CVPR (2010)

7. Chang, C.C., Lin, C.J.: Libsvm: A library for support vector machines. ACM Transactions on Intelligent Systems and Technology 2, 27:1-27:27 (2011)

8. Chatfield, K., Lempitsky, V., Vedaldi, A., Zisserman, A.: The devil is in the details: an evaluation of recent feature encoding methods. In: BMVC (2011)

9. Cheng, H., Yan, X., Han, J., Hsu, C.W.: Discriminative frequent pattern analysis for effective classification. In: ICDE, pp. 716 -725 (2007). DOI 10.1109/ICDE.2007.367917

10. Chum, O., Perdoch, M., Matas, J.: Geometric min-hashing: Finding a (thick) needle in a haystack. In: CVPR (2009). DOI 10.1109/CVPR.2009.5206531

11. Cinbis, R.G., Verbeek, J., Schmid, C.: Image categorization using fisher kernels of noniid image models. In: CVPR (2012)

12. Csurka, G., Dance, C.R., Fan, L., Willamowski, J., Bray, C.: Visual categorization with bags of keypoints. In: Work. on Statistical Learning in CV, pp. 1-22 (2004)

13. Dalal, N., Triggs, B.: Histograms of oriented gradients for human detection. In: IEEE Conference on Computer Vision and Patternn Recognition (CVPR) (2005)

14. Endres, I., Shih, K.J., Jiaa, J., Hoiem, D.: Learning collections of part models for object recognition. In: The IEEE Conference on Computer Vision and Pattern Recognition (CVPR) (2013)

15. Everingham, M., Van Gool, L., Williams, C.K.I., Winn, J., Zisserman, A.: The PASCAL Visual Object Classes Challenge 2007 Results. http://www.pascal-network.org/ challenges/VOC/ voc2007/workshop/ index.html (2007)

16. Farhadi, A., Endres, I., Hoiem, D., Forsyth, D.: Describing objects by their attributes. In: CVPR, pp. $1778-1785$ (2009). DOI 10.1109/CVPR.2009.5206772

17. Fernando, B., Fromont, E., Muselet, D., Sebban, M.: Discriminative feature fusion for image classification. In: CVPR (2012)

18. Fernando, B., Fromont, É., Tuytelaars, T.: Effective use of frequent itemset mining for image classification. In: ECCV, Lecture Notes in Computer Science, vol. 7572, pp. 214-227. Springer (2012) 
19. Fernando, B., Tuytelaars, T.: Mining multiple queries for image retrieval: On-the-fly learning of an object-specific mid-level representation. In: ICCV (2013)

20. Gilbert, A., Illingworth, J., Bowden, R.: Fast realistic multi-action recognition using mined dense spatio-temporal features. In: ICCV, pp. $925-931$ (2009). DOI 10.1109/ICCV.2009.5459335

21. Jaakkola, T., Haussler, D.: Exploiting generative models in discriminative classifiers. In: NIPS, pp. 487-493 (1998)

22. Juneja, M., Vedaldi, A., Jawahar, C.V., Zisserman, A.: Blocks that shout: Distinctive parts for scene classification. In: CVPR (2013)

23. Kim, S., Jin, X., Han, J.: Disiclass: discriminative frequent pattern-based image classification. In: Tenth Int. Workshop on Multimedia Data Mining (2010). DOI http://doi.acm.org/10.1145/1814245.1814252. URL http://doi.acm.org/10.1145/1814245.1814252

24. Lazebnik, S., Schmid, C., Ponce, J.: Beyond bags of features: Spatial pyramid matching for recognizing natural scene categories. In: CVPR, pp. 2169-2178 (2006)

25. Lee, A.J., Liu, Y.H., Tsai, H.M., Lin, H.H., Wu, H.W.: Mining frequent patterns in image databases with 9d-spa representation. Journal of Systems and Software 82(4), 603 - 618 (2009). DOI DOI: 10.1016/j.jss.2008.08.028. URL http://www.sciencedirect.com/science/article/pii/S0164121208002069. Special Issue: Selected papers from the 2008 IEEE Conference on Software Engineering Education and Training (CSEET08)

26. Lee, Y.J., Efros, A.A., Hebert, M.: Style-aware mid-level representation for discovering visual connections in space and time. In: International Conference on Computer Vision (2013)

27. Ling, H., Soatto, S.: Proximity distribution kernels for geometric context in category recognition. In: ICCV (2007)

28. Liu, D., Hua, G., Viola, P., Chen, T.: Integrated feature selection and higher-order spatial feature extraction for object categorization. In: CVPR (2008)

29. Lowe, D.G.: Object recognition from local scale-invariant features. In: ICCV, pp. 11501157 (1999)

30. Nilsback, M.E., Zisserman, A.: Automated flower classification over a large number of classes. In: ICVGIP, pp. 722 -729 (2008). DOI 10.1109/ICVGIP.2008.47

31. Nowozin, S., Tsuda, K., Uno, T., Kudo, T., Bakir, G.: Weighted substructure mining for image analysis. In: CVPR (2007). DOI 10.1109/CVPR.2007.383171. URL http://www.nowozin.net/sebastian/gboost/

32. Opelt, A., Fussenegger, M., Pinz, A., Auer, P.: Weak hypotheses and boosting for generic object detection and recognition. In: ECCV, pp. 71-84 (2004)

33. Perronnin, F., Sánchez, J., Mensink, T.: Improving the fisher kernel for large-scale image classification. In: ECCV, pp. 143-156 (2010). URL http://dl.acm.org/citation.cfm?id=1888089.1888101

34. Quack, T., Ferrari, V., Gool, L.V.: Video mining with frequent itemset configurations. In: CIVR, pp. 360-369 (2006)

35. Quack, T., Ferrari, V., Leibe, B., Van Gool, L.: Efficient mining of frequent and distinctive feature configurations. In: ICCV (2007)

36. Rematas, K., Fritz, M., Tuytelaars, T.: The pooled nbnn kernel: Beyond image-to-class and image-to-image. In: ACCV, vol. 7724, pp. 176-189 (2012)

37. Savarese, S., Winn, J., Criminisi, A.: Discriminative object class models of appearance and shape by correlatons. In: CVPR (2006)

38. Shahbaz Khan, F., van de Weijer, J., Vanrell, M.: Top-down color attention for object recognition. In: ICCV, pp. 979 -986 (2009)

39. Sharma, G., Jurie, F., Schmid, C.: Expanded parts model for human attribute and action recognition in still images. In: CVPR (2013)

40. Simonyan, K., Vedaldi, A., Zisserman, A.: Deep fisher networks for large-scale image classification. In: Advances in Neural Information Processing Systems (2013)

41. Singh, S., Gupta, A., Efros, A.: Unsupervised discovery of mid-level discriminative patches. In: ECCV (2012)

42. Sivic, J., Zisserman, A.: Video Google: A text retrieval approach to object matching in videos. In: ICCV, vol. 2, pp. 1470-1477 (2003) 
43. Sivic, J., Zisserman, A.: Video data mining using configurations of viewpoint invariant regions. In: CVPR (2004). DOI 10.1109/CVPR.2004.1315071

44. Tuytelaars, T., Fritz, M., Saenko, K., Darrell, T.: The nbnn kernel. In: ICCV, pp. 1824-1831 (2011)

45. Uno, T., Asai, T., Uchida, Y., Arimura, H.: Lcm: An efficient algorithm for enumerating frequent closed item sets. In: FIMI (2003). URL http://fimi.ua.ac.be/src/

46. van de Weijer, J., Schmid, C.: Applying color names to image description. In: ICIP, pp. 493-496 (2007)

47. Xie, N., Ling, H., Hu, W., Zhang, X.: Use bin-ratio information for category and scene classification. In: CVPR, pp. 2313 -2319 (2010). DOI 10.1109/CVPR.2010.5539917

48. Yan, X., Cheng, H., Han, J., Xin, D.: Summarizing itemset patterns: a profile-based approach. In: ACM SIGKDD (2005)

49. Yang, Y., Newsam, S.: Spatial pyramid co-occurrence for image classification. In: ICCV (2011)

50. Yao, B., Fei-Fei, L.: Grouplet: A structured image representation for recognizing human and object interactions. In: CVPR (2010)

51. Yimeng Zhang, T.C.: Efficient kernels for identifying unbounded-order spatial features. In: CVPR (2009)

52. Yuan, J., Luo, J., Wu, Y.: Mining compositional features for boosting. In: CVPR (2008). DOI 10.1109/CVPR.2008.4587347

53. Yuan, J., Wu, Y., Yang, M.: Discovery of collocation patterns: from visual words to visual phrases. In: CVPR (2007). DOI 10.1109/CVPR.2007.383222

54. Yuan, J., Yang, M., Wu, Y.: Mining discriminative co-occurrence patterns for visual recognition. In: CVPR, pp. 2777 -2784 (2011). DOI 10.1109/CVPR.2011.5995476

55. Yun, U., Leggett, J.J.: Wfim: Weighted frequent itemset mining with a weight range and a minimum weight. In: SDM'05 (2005) 